\title{
Comparative Evaluation of Efficacy and Safety of Intramuscular Butorphanol vs Epidural Butorphanol as Postoperative Analgesia in Patients Undergoing Lower Abdominal and Lower Limb Surgeries: A Randomized-controlled Study
}

\author{
Chaula Doshi ${ }^{1}$, Varsha H Vyas ${ }^{2}$, Bobby Kurian ${ }^{3}$, Surekha S Patil ${ }^{4}$, Deepakkumar G Langade ${ }^{5}$
}

\begin{abstract}
Context: Intramuscular (IM) butorphanol can be a convenient postoperative analgesia compared to epidural (EP) route.

Aims: Compare the efficacy and safety of IM vs EP butorphanol (1 mg/2 mg) as postoperative analgesia.

Settings and design: A randomized-controlled study.

Materials and methods: This study was conducted on 120 adult patients of either sex in the age group of 20-60 years, undergoing lower abdominal or lower limb surgeries (American Society of Anesthesiologists [ASA] class-I/II). Patients were randomized to butorphanol intramuscularly (IM group) or EP injection (EP group). Each group had two equal subgroups where patients received either $1 \mathrm{mg}$ or $2 \mathrm{mg}$ by the respective route. The primary outcomes were onset of analgesia, peak time of analgesia, and duration of analgesia. Pain was assessed on a $0-10$ visual analog scale (VAS), and vital parameters were assessed at baseline and after 10, 20, 30, 60, 90, 120, and 180 minutes. Clinical safety was assessed by the adverse events.

Results: The duration of analgesia provided by both the routes was comparable (293.0 minutes IM and 270.25 minutes EP). However, duration of analgesia was longer $(p, 0.043)$ with $2 \mathrm{mg}$ compared to $1 \mathrm{mg}$. Similarly, the onset of analgesia, pain scores, and peak time of analgesia were similar $(p>0.05)$ in the two groups. No differences in the hemodynamic parameters and respiratory rate were observed in the two groups. Higher incidence of all the side effects (sedation, somnolence, pruritus) was observed with IM administration. All side effects were clinically insignificant. Conclusion: Intramuscular butorphanol is as effective as an EP for the management of immediate postoperative pain.

Keymessages: Intramuscular analgesics could be more convenient and safer compared to EP administration. Butorphanol administered by the IM route is as effective and safe compared to EP administration.

Keywords: Analgesia, Butorphanol, Epidural, Intramuscular, Postoperative.

Research and Innovation in Anesthesia (2019): 10.5005/jp-journals-10049-0065
\end{abstract}

\section{INTRODUCTION}

Pain is an unpleasant sensory and emotional experience associated with actual or potential tissue damage or described in terms of such damage. ${ }^{1}$ Postoperative pain causes physiological and psychological phenomena and is a major concern. Effective pain control is, therefore vital for early mobilization and postoperative discharge. ${ }^{2}$ The multidimensional effects of pain often require many modes of therapy, including pharmacological treatment, behavioral and psychological interventions, psychiatric treatments, and family and spiritual support. Postoperative pain relief requires multimodal therapy, and of this pharmacological treatment, is the most important mode.

Opioids are the cornerstone of acute pain relief, and they are powerful, centrally acting agents, which also have peripheral effects. Opioids inhibit the neuronal action potentials not only at nociceptive but also specific sensory, somatosensory, and autonomic neurons. ${ }^{3}$ Epidural administration of narcotic analgesics is the standard therapy for postoperative analgesia in EP anesthesia. ${ }^{4}$ However, they are associated with troublesome effects like respiratory depression, urinary retention, pruritus, nausea, and vomiting. ${ }^{5,6}$ Butorphanol is a lipid-soluble narcotic with strong K-receptor agonist and weak $\mu$-receptor agonist/antagonist activity.
${ }^{1}$ Department of Anesthesia, KJ Somaiya Medical College and Research Centre, Mumbai, Maharashtra, India

${ }^{2,4}$ Department of Anesthesia, DY Patil University School of Medicine, Navi Mumbai, Maharashtra, India

${ }^{3}$ Sabah Al Ahmed Urology Centre, Shuwaikh Medical Area, Kuwait

${ }^{5}$ Department of Pharmacology, DY Patil University School of Medicine, Navi Mumbai, Maharashtra, India

Corresponding Author: Varsha H Vyas, Department of Anesthesia, DY Patil University School of Medicine, Navi Mumbai, Maharashtra, India, Phone: +91 9819583232, e-mail: varsha.vyas011@gmail.com

How to cite this article: Doshi C, Vyas VH, Kurian B, et al. Comparative Evaluation of Efficacy and Safety of Intramuscular Butorphanol vs Epidural Butorphanol as Postoperative Analgesia in Patients Undergoing Lower Abdominal and Lower Limb Surgeries: A Randomized-controlled Study. Res Inno in Anesth 2019;4(1):1-6.

Source of support: Nil

Conflict of interest: None

These have been frequently used for postoperative analgesia. ${ }^{7}$ It was first available in 2002 in India and is considered safer than pure agonist opioids because of its ceiling effect on respiratory

o The Author(s). 2019 Open Access This article is distributed under the terms of the Creative Commons Attribution 4.0 International License (https://creativecommons. org/licenses/by-nc/4.0/), which permits unrestricted use, distribution, and non-commercial reproduction in any medium, provided you give appropriate credit to the original author(s) and the source, provide a link to the Creative Commons license, and indicate if changes were made. The Creative Commons Public Domain Dedication waiver (http://creativecommons.org/publicdomain/zero/1.0/) applies to the data made available in this article, unless otherwise stated. 
depression, lower addiction potential, lesser cardiovascular and gastrointestinal (postoperative nausea and vomiting) side effects, lesser pruritus or urinary retention as compared to morphine. Due to the complexity of EP administration and its associated risks, it would be convenient and safe to use IM injection as postoperative analgesia. The primary objective was to compare the efficacy of IM butorphanol (1 mg/2 mg) vs EP butorphanol as postoperative analgesia in patients undergoing lower abdominal and lower limb surgeries. Secondary objective was the safety comparison of IM vs EP administration of butorphanol. It was hypothesized that the IM butorphanol would be as effective and safe as EP injection as a postoperative analgesic.

\section{Materials and Methods}

This randomized-controlled study was carried out after obtaining approval from the Institutional Ethics Committee (IEC). The study was conducted in accordance with the principles of the Declaration of Helsinki (World Medical Association) and Good Clinical Practice (GCP) guidelines issued by the Indian Council of Medical Research (ICMR) and Drugs Controller General, India (DCGI), Government of India. All the patients were explained about the study procedures, and informed consent was obtained from all the patients.

Patients of either gender, between 20 years and 60 years of age, patients of ASA stage-I and stage-II, undergoing lower abdominal or lower limb surgeries were enrolled after obtaining written informed consent. Patients in whom regional anesthesia is contraindicated, patients with allergies to the study medication, and patients with opioid dependence were excluded.

A total of 120 study eligible patients were randomized to receive intramuscularly $(n=60)$ or EP $(n=60)$ injections of butorphanol (Aristo, India). Since there are no published studies comparing IM and EP butorphanol as postoperative analgesia, this was a pilot study, and sample size was not based on any estimations and assumptions (Flowchart 1).
A stratified randomization was done using PC based predetermined randomization schedule. The randomization was prepared by independent personnel and was concealed in sealed separate envelopes for each study participant. The study team was blinded for the randomization, and allocation was done only after the eligible participant was enrolled and assigned a study serial number. After enrolment, the sealed envelope was opened by the study team members to reveal the treatment allocation for the participant. The study was open after randomization was done. Thirty patients in each IM group and EP group received either $1 \mathrm{mg}$ or $2 \mathrm{mg}$ butorphanol as per the randomization. Thus, there were two study groups with two subgroups each based on dose.

The EP injection was administered as $10 \mathrm{~mL}$ (diluted in $0.9 \%$ sodium chloride) volume containing $1 \mathrm{mg} / 2 \mathrm{mg}$, whereas the IM injection was given in a $1 \mathrm{~mL}$ injection containing $1 \mathrm{mg} / 2 \mathrm{mg}$. Butorphanol administration was done postoperatively when the effect of regional analgesia wore off, and the patient complained of severe pain with a VAS score of $\geq 7$ for pain. This time-point was considered as the baseline. Clinical assessments were done for surgical site pain and vital parameters. Assessment of pain was done using a 0-10 linear VAS, where " 0 " represents no pain, and " 10 " represents severe intolerable pain. ${ }^{8}$ At baseline, all recruited patients received injection butorphanol intramuscularly (IM) or epidurally (ED) as per the randomization. The primary outcomes were onset of analgesia, peak time of analgesia, and duration of analgesia. Secondary outcome was safety based on vital parameters and adverse effects. All vital parameters and VAS scores were noted at baseline and then after 10, 20,30,60, 90, 120, and 180 minutes. Patients were monitored every 10 minutes, and pain score and vital parameters recorded at each time-period. Vital parameters recorded were pulse rate, blood pressure [mean arterial pressure (MAP)], and respiratory rate. Clinical safety was assessed based on the sedation and other adverse effects reported by the patients.

The baseline descriptive data for the two groups were compared using a one-way analysis of variance (ANOVA), whereas

Flowchart 1: COSORT flow diagram

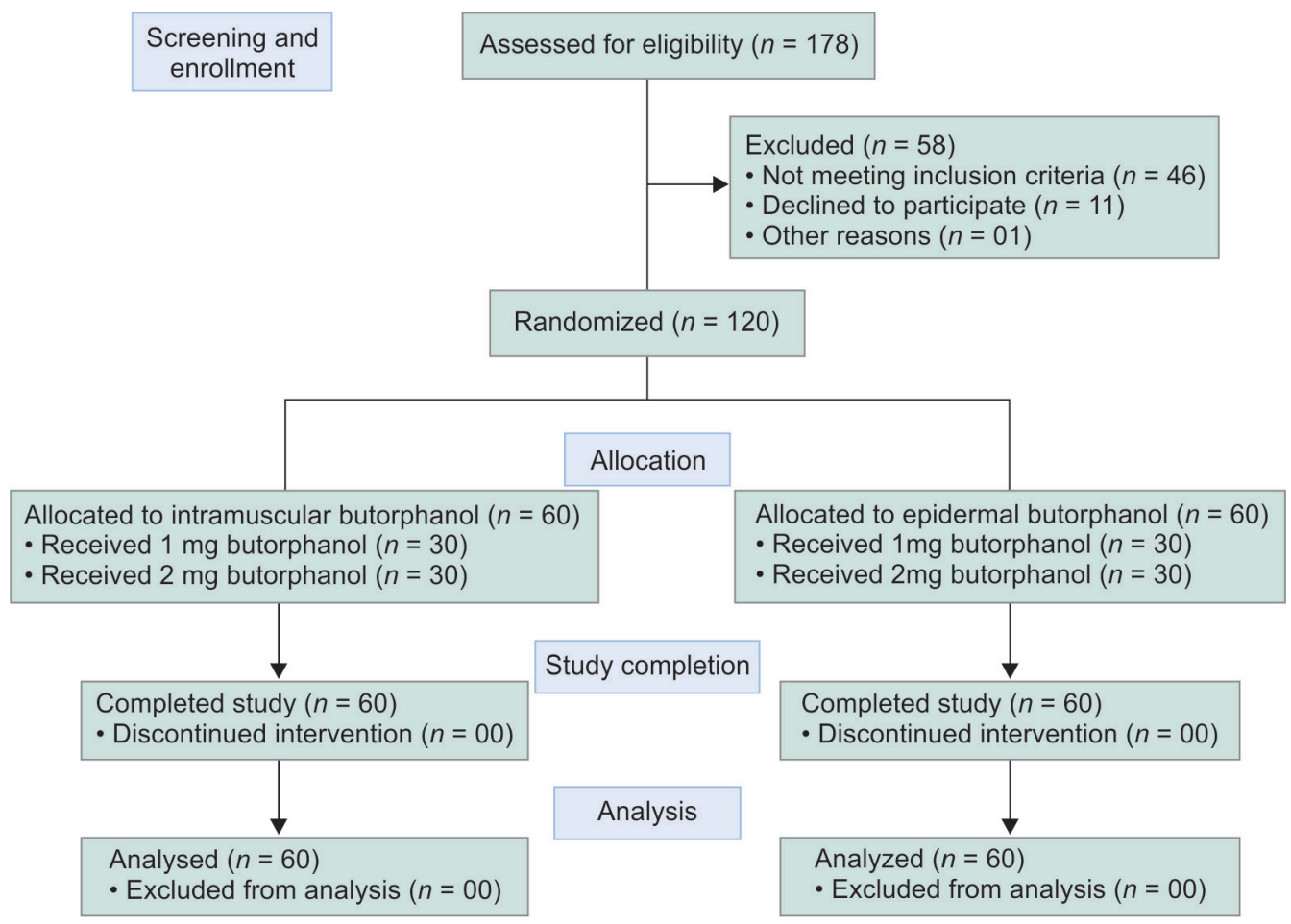


the discrete data were compared using the Chi-square test. Analysis of the primary outcomes (onset of analgesia, peak time of analgesia, and duration of analgesia) using two-way ANOVA with the route of administration as the first factor and dose used as the second factor. General linear model (GLM) procedure (repeat measures ANOVA) with time and route of administration as the factors were used for the analysis of the changes in the pain scores and vital parameters over 3 hours after dosing between the two groups.

\section{Results}

Table 1 shows the demography and baseline data of patients enrolled in the two groups. The two groups were comparable $(p,>0.05)$ with respect to the age, body mass index (BMI), ASA physical status class, gender distribution and all baseline vital parameters except systolic blood pressure, which was higher $(p, 0.005)$ in the IM group.

Table 2 shows the study outcomes in the two groups. The onset of analgesia, peak time of analgesia, and duration of analgesia were similar in both IM and EP groups $(p>0.05)$. Also, there were no differences between the two doses $1 \mathrm{mg}$ and $2 \mathrm{mg}$ for the duration of surgery, onset of analgesia, and peak time of analgesia. Large effect sizes were observed for the peak time of analgesia (1.256, $88 \%)$ and duration of analgesia (2.30,98\%), where very small effect size was observed for the onset of analgesia (2.30,58\%). However, the duration of analgesia was littler longer $(p, 0.043)$ with $2 \mathrm{mg}$ dose compared to $1 \mathrm{mg}$ dose. The mean VAS scores for pain (Fig. 1) were similar in the two groups at different time intervals $(p, 0.770)$.

1 ne MAP, pulse rate, and respiratory rates are presented in Figures 2 to 4 , respectively. The hemodynamic parameters and respiratory rates were similar in the two groups $(p>0.05)$.

Table 3 presents the adverse effects reported by the patients during the study period. Adverse effects observed were sedation, somnolence, nausea/vomiting, and pruritus. The incidence of all events was more with IM butorphanol ( $p<0.0001)$. Surprisingly, the incidence of events was greater, with $1 \mathrm{mg}$ dose compared to $2 \mathrm{mg}$ butorphanol. However, none of the side effects was clinically significant, requiring any intervention.

\section{Discussion}

Despite a better understanding of pain pathophysiology and more treatment options available, postoperative pain still is a challenge and remains poorly treated. ${ }^{9}$ Postoperative pain is an important social, medical, and economic concern as it increases the morbidity. Effective pain control is hence essential for the optimal care during postoperative period as these patients suffer from considerable pain, and it has been recognized as a prime concern for anesthesiologists. ${ }^{2}$ Epidural route is used extensively for postoperative pain control, and EP opioids have been administered commonly to relieve anxiety and reduce pain associated with surgery. Combined EP anesthesia with postoperative EP analgesia is

Table 1: Demography and baseline data of patients enrolled

\begin{tabular}{|c|c|c|c|c|c|c|c|c|}
\hline & \multicolumn{3}{|c|}{ Intramuscular $(n=60)$} & \multicolumn{3}{|c|}{ Epidural $(n=60)$} & \multicolumn{2}{|c|}{ ANOVA } \\
\hline & Mean & $S D$ & 95\% C.I. & Mean & $S D$ & 95\% C.I. & $F$ & $p$ \\
\hline Age (years) & 43.80 & 13.38 & $40.34-47.26$ & 46.88 & 13.26 & $43.46-50.31$ & 1.608 & 0.207 \\
\hline BMI (kg/sq m) & 22.88 & 4.20 & $21.79-23.96$ & 22.70 & 4.55 & $21.52-23.87$ & 0.049 & 0.824 \\
\hline VAS score & 8.18 & 0.89 & $7.95-8.40$ & 8.27 & 0.92 & $8.03-8.50$ & 0.309 & 0.579 \\
\hline $\mathrm{SBP}(\mathrm{mm} \mathrm{Hg})$ & 132.88 & 12.55 & $129.64-136.12$ & 125.65 & 15.23 & $121.72-129.58$ & 8.063 & 0.005 \\
\hline $\mathrm{DBP}(\mathrm{mm} \mathrm{Hg})$ & 80.82 & 9.02 & $78.49-83.15$ & 77.88 & 9.89 & $75.33-80.44$ & 2.881 & 0.092 \\
\hline MAP (mm Hg) & 98.17 & 9.18 & $95.80-100.54$ & 93.81 & 10.55 & $91.08-96.53$ & 5.850 & 0.017 \\
\hline Pulse rate (per minute) & 88.18 & 10.69 & $85.42-90.94$ & 87.43 & 11.82 & $84.38-90.49$ & 0.133 & 0.716 \\
\hline RR (per minute) & 16.30 & 2.05 & $15.77-16.83$ & 16.58 & 1.74 & $16.13-17.03$ & 0.665 & 0.416 \\
\hline Surgery duration (minute) & 130.42 & 4.45 & $121.60-139.24$ & 135.33 & 4.45 & $126.51-144.15$ & 0.609 & 0.437 \\
\hline \multicolumn{9}{|l|}{ Gender } \\
\hline Male & 34 & $56.67 \%$ & & 25 & $41.67 \%$ & & & 0.072 \\
\hline Female & 26 & $43.33 \%$ & & 35 & $58.33 \%$ & & & \\
\hline \multicolumn{9}{|l|}{ BMI category } \\
\hline Underweight & 8 & $13.33 \%$ & & 9 & $15.00 \%$ & & & 0.900 \\
\hline Normal weight & 37 & $61.67 \%$ & & 33 & $55.00 \%$ & & & \\
\hline Overweight & 12 & $20.00 \%$ & & 14 & $23.33 \%$ & & & \\
\hline Obese & 3 & $5.00 \%$ & & 4 & $6.67 \%$ & & & \\
\hline \multicolumn{9}{|l|}{ ASA stage } \\
\hline Stage I & 32 & $53.33 \%$ & & 32 & $53.33 \%$ & & & 1.000 \\
\hline Stage II & 28 & $46.67 \%$ & & 28 & $46.67 \%$ & & & \\
\hline \multicolumn{9}{|l|}{ Surgery } \\
\hline Appendicectomy & 35 & $58.33 \%$ & & 32 & $53.33 \%$ & & & 0.870 \\
\hline Amputation & 4 & $6.67 \%$ & & 3 & $5.00 \%$ & & & \\
\hline Hernia & 21 & $35.00 \%$ & & 25 & $41.67 \%$ & & & \\
\hline \multicolumn{9}{|l|}{ Butorphanol dose } \\
\hline $1 \mathrm{mg}$ & 30 & $50.00 \%$ & & 30 & $50.00 \%$ & & & 1.000 \\
\hline $2 \mathrm{mg}$ & 30 & $50.00 \%$ & & 30 & $50.00 \%$ & & & \\
\hline
\end{tabular}

ASA, American Society of Anesthesiologists; BMI, body mass index; DBP, diastolic blood pressure; MAP, mean arterial pressure; RR, respiratory rate; SBP, systolic blood pressure; VAS, visual analog scale 
Table 2: Study outcomes in the two groups

\begin{tabular}{|c|c|c|c|c|c|c|c|c|c|}
\hline & \multicolumn{3}{|c|}{ Intramuscular } & \multicolumn{3}{|c|}{ Epidural } & \multicolumn{3}{|c|}{ Two-way ANOVA } \\
\hline & Mean & SEM & $95 \% \mathrm{Cl}$ & Mean & SEM & $95 \% \mathrm{Cl}$ & $F$ & $p$ (route) & $p$ (dose) \\
\hline \multicolumn{10}{|c|}{ Onset of analgesia (minute) } \\
\hline $1 \mathrm{mg}$ & 17.83 & 0.99 & 15.87-19.79 & 22.53 & 0.99 & $20.57-24.49$ & & & \\
\hline $2 \mathrm{mg}$ & 23.50 & 0.99 & $21.54-25.46$ & 17.23 & 0.99 & 15.27-19.19 & & & \\
\hline Total & 20.18 & 0.78 & $18.63-21.73$ & 20.37 & 0.78 & 18.82-21.92 & 0.027 & 0.869 & 0.480 \\
\hline \multicolumn{10}{|c|}{ Peak time of analgesia (minute) } \\
\hline $1 \mathrm{mg}$ & 53.60 & 3.61 & $46.46-60.74$ & 78.03 & 3.61 & $70.89-85.17$ & & & \\
\hline $2 \mathrm{mg}$ & 80.33 & 3.61 & $73.19-87.47$ & 43.17 & 3.61 & $36.02-50.31$ & & & \\
\hline Total & 65.82 & 3.24 & $59.40-72.23$ & 61.75 & 3.24 & $55.33-68.17$ & 0.788 & 0.377 & 0.167 \\
\hline \multicolumn{10}{|c|}{ Duration of analgesia (minute) } \\
\hline $1 \mathrm{mg}$ & 266.33 & 13.87 & $238.85-293.81$ & 274.17 & 13.87 & $246.68-301.64$ & & & \\
\hline $2 \mathrm{mg}$ & 268.33 & 13.87 & $240.85-295.81$ & 317.67 & 13.87 & $290.18-345.14$ & & & \\
\hline Total & 270.25 & 9.86 & $250.72-289.78$ & 293.00 & 9.86 & $273.47-68.17$ & 2.661 & 0.106 & 0.043 \\
\hline
\end{tabular}

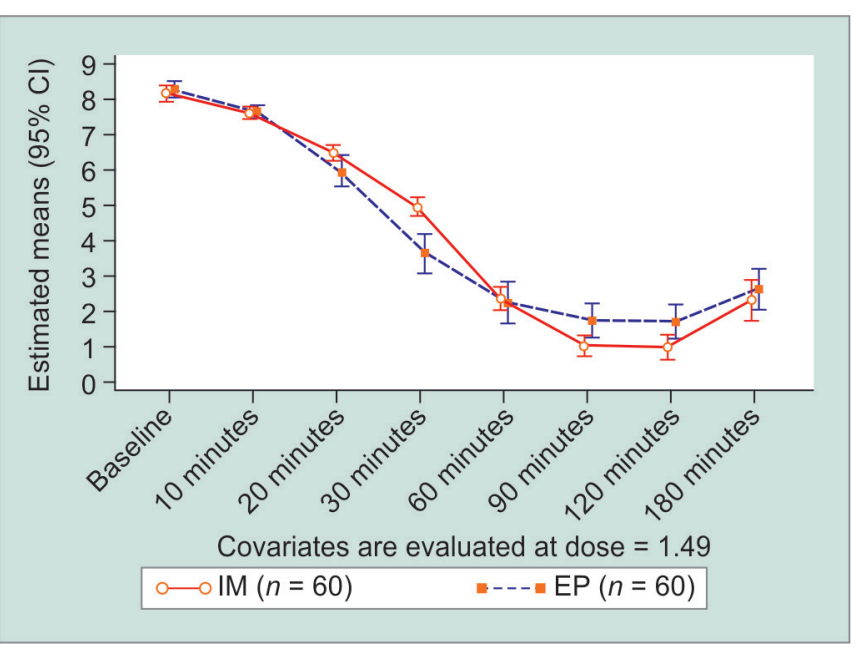

Fig. 1: Pain scores (visual analog scale)

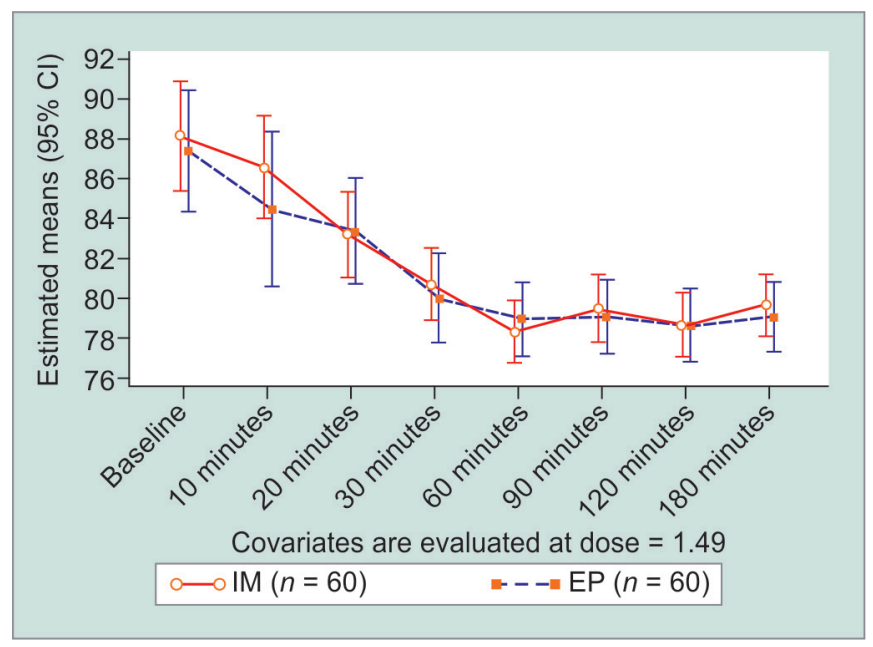

Fig. 3: Pulse rate (per minute)

commonly practiced due to better outcomes after major surgery. ${ }^{10}$ Meta-analyses suggest that EP anesthesia and postoperative analgesia may facilitate earlier recovery and improved outcomes by reducing the incidence of postoperative complications after major surgery.

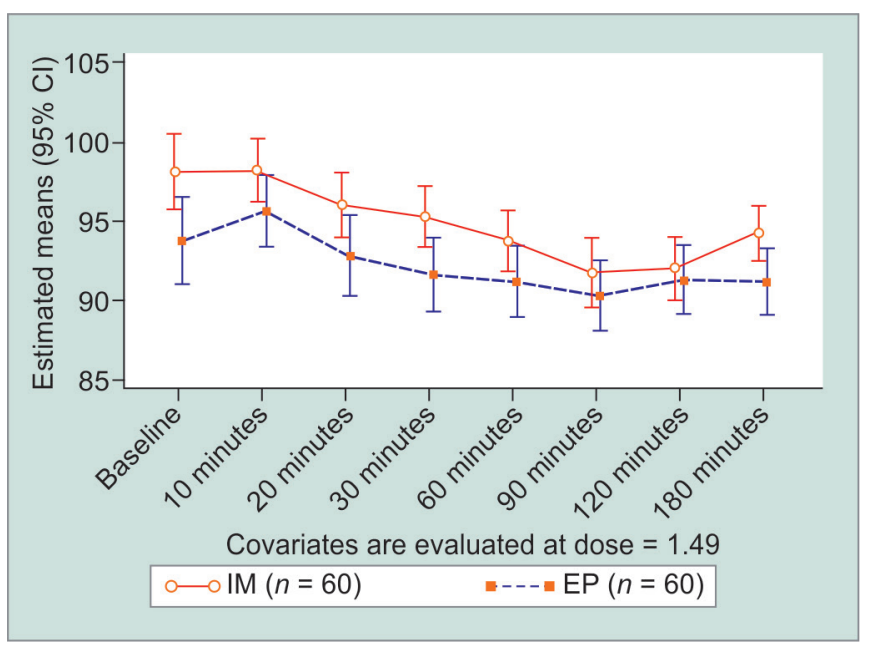

Fig. 2: Mean arterial pressure $(\mathrm{mm} \mathrm{Hg})$

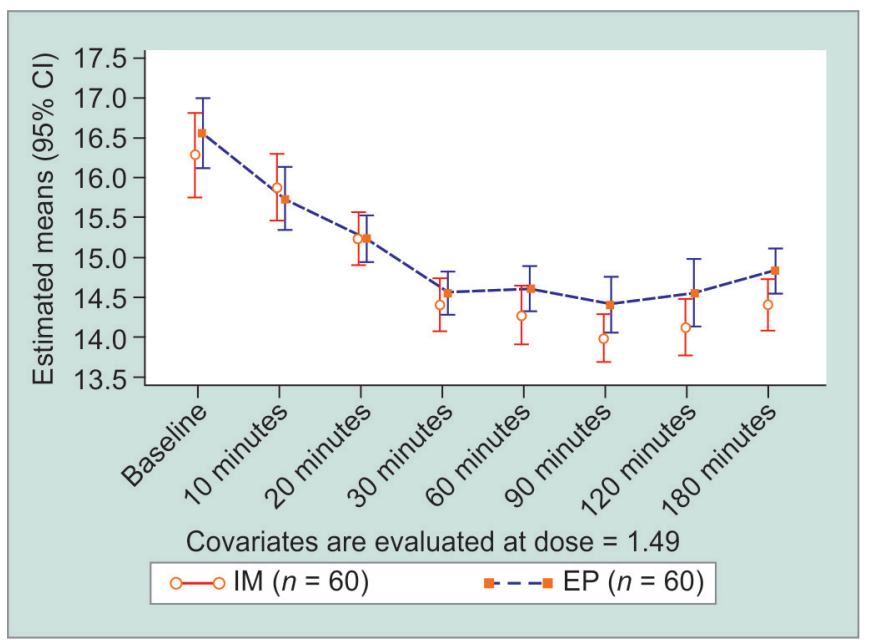

Fig. 4: Respiratory rate (per minute)

Postoperative opioid use is very less probably due to a lack of knowledge, fear of adverse effects, and addictive potential. However, butorphanol is considered safe with fewer adverse effects compared to pure agonist opioids and is frequently used for postoperative analgesia. ${ }^{7}$ In the dose of 1 or $2 \mathrm{mg}$, butorphanol 
Lower Abdominal and Lower Limb Surgeries: A Randomized-controlled Study

Table 3: Adverse events reported by patients in the two groups

\begin{tabular}{|c|c|c|c|c|c|c|c|}
\hline & \multicolumn{3}{|c|}{ Intramuscular $(n=60)$} & \multicolumn{3}{|c|}{ Epidural $(n=60)$} & \multirow[b]{2}{*}{$p\left(\chi^{2}\right.$ test $)$} \\
\hline & $1 \mathrm{mg}(n=30)$ & $2 m g(n=30)$ & Total $(n=60)$ & $1 m g(n=30)$ & $2 m g(n=30)$ & Total $(n=60)$ & \\
\hline Sedation & 10 & 9 & $19(31.7 \%)$ & 3 & 7 & $10(16.7 \%)$ & \\
\hline Somnolence & 9 & 0 & $9(15.0 \%)$ & 0 & 0 & $0(-)$ & \\
\hline Nausea/vomiting & 4 & 0 & $4(6.7 \%)$ & 0 & 0 & $0(-)$ & \\
\hline Pruritus & 2 & 0 & $2(3.3 \%)$ & 0 & 1 & $1(1.7 \%)$ & \\
\hline Total events & 25 & 9 & $34(56.7 \%)$ & 3 & 8 & $11(18.3 \%)$ & \\
\hline Total patients & 25 & 9 & $34(56.7 \%)$ & 3 & 8 & $11(18.3 \%)$ & $<0.0001$ \\
\hline
\end{tabular}

has been found to be a safe and effective adjuvant in combination with bupivacaine for labor analgesia. ${ }^{11}$ Abboud et al. concluded that adding small doses of butorphanol $(1 \mathrm{mg})$ to EP lidocaine during labor is effective and safe. ${ }^{12}$ Butorphanol is also useful in combination with $0.1 \%$ bupivacaine during EP analgesia for labor as it provides excellent pain relief, prolongs the duration of action with simultaneously decreasing the need for analgesic requirement, thereby reducing the total local anesthetic. ${ }^{13}$

Our study was a randomized controlled study comparing IM butorphanol vs EP butorphanol for their efficacy as a postoperative analgesic. We studied both male and female patients ported for abdominal and orthopedic surgery and experiencing postoperative pain. Our study included patients with both ASA stage-I and stage-II and all BMI categories. The anesthetic procedures and preanesthetic medications were similar in all patients, and a standard protocol was followed. We had used equianalgesic doses for EP and IM route of administration. We also studied the effect of butorphanol dose $1 \mathrm{mg} / 2 \mathrm{mg}$ on the analgesic efficacy. We found no differences between the pain scores when butorphanol was administered by IM or EP route. Also, the dose of $1 \mathrm{mg}$ or $2 \mathrm{mg}$ had similar analgesic effects ( $p>0.05)$ on surgery duration, onset of analgesia, and peak time of analgesia. However, the duration of analgesia was longer with a higher dose of butorphanol $(p, 0.043)$.

In a recent randomized, double-blind study comparing IM butorphanol vs pentazocine for postoperative pain relief, butorphanol was reported to be more effective and safer analgesia compared to pentazocine for postoperative pain management in gynecologic lower abdominal surgery. ${ }^{14}$ Here, both nalbuphine and butorphanol administered intramuscularly provided better analgesia, rapid onset, and longer duration of analgesia compared to pentazocine. Also, the incidence of nausea and vomiting was also low as against pentazocine. ${ }^{14}$ Butorphanol is reported to be more effective analgesia with lesser side effects as compared to fentanyl in a comparative study in lower abdominal surgeries. ${ }^{15}$ Butorphanol is also used as EP adjuvants in abdominal hysterectomy under intrathecal levobupivacaine anesthesia. ${ }^{16}$

We used both $1 \mathrm{mg}$ and $2 \mathrm{mg}$ doses of butorphanol by both IM and EP route. A comparative study of EP butorphanol with bupivacaine vs bupivacaine monotherapy for analgesia reported that butorphanol $2 \mathrm{mg}$ and $4 \mathrm{mg}$ can be used as a safe and effective adjuvant to EP bupivacaine in patients undergoing lower limb orthopedic surgeries. ${ }^{17}$

The prime function of the anesthesiologist is to relieve pain. As anesthesiologist has the necessary technical skill and pharmacological knowledge, they are in an ideal position to treat pain in many situations; such as perioperative pain, chronic pain, labor analgesia, etc. In the postoperative period, when the effect of anesthesia wears off, the tissue injury persists, and the pain-producing substances that are liberated during operations greatly reduce the normal high threshold of nociceptors so that innocuous stimulation produces great pain. Opioids have been used for many years as an important constituent for providing adequate analgesia. Previously sublingual, IM, and intravenous routes were used, but recently, intrathecal and EP administration of opioids has been widely studied. Epidural route is preferred over intrathecal route because it is less invasive, low risk of infection, lack of postspinal headache, and lower incidence of side effects. Traditionally EP bupivacaine was used in lower concentrations for postoperative analgesia.

Butorphanol tartrate is a synthetically derived agonistantagonist opioid analgesic. It is 7-10 times more potent than morphine and has been employed successfully in Western countries since its launch in 1978 for the relief of postoperative pain, preanesthetic medication and in balanced anesthesia. Butorphanol is considered safer than morphine because of its dose-related ceiling effect on respiratory depression, lesser side effects, i.e., nausea/vomiting, pruritus or urinary retention, and lower addiction potential. However, it produces a significant degree of sedation.

The hemodynamic parameters and the respiratory rates were similar with both IM and EP route of butorphanol administration $(p>0.05)$. The common adverse events reported in our study with butorphanol were sedation, somnolence, nausea/vomiting, and pruritus. The adverse effects with IM butorphanol are higher compared to EP butorphanol, which also includes sedation. In our study, we considered patients undergoing lower abdominal and lower limb surgeries under general anesthesia or combined spinal-EP anesthesia. One hundred and twenty adult patients (20-60 years) of either sex of ASA stage-I or stage-II were recruited in the study. The mean of onset analgesia of group IM was 20.18, and of the group, EP was 20.37, onset analgesia was significantly faster in EP group. Peak of analgesia is taken in the time intervals, meantime of peak of analgesia in EP was 61.75, and of IM was 65.82, the peak analgesia achieved in EP group was faster than IM group. The pilot nature of the study was the main limitation of the study apart from the small sample size for the onset of analgesia as observed by the small effect size. Based on our observations, we further plan to conduct a large-scale study. Also, the study could have been done in other types of surgical procedures to widen the scope of use of IM butorphanol as a postoperative analgesic. The main strength of the study was the randomized controlled study design.

Intramuscular butorphanol is as effective as an EP injection for the management of immediate postoperative pain. However, it provides a slower onset of analgesia compared to EP administration. Also, butorphanol tartrate given epidurally in a dose of $2 \mathrm{mg}$ gives a faster onset and better effect for a longer duration. 


\section{References}

1. Bond MR, Bonica JJ, Boyd DB, et al. Classification of chronic pain. Pain 1979;6:249-252.

2. Revar B, Patel V, Patel B, et al. A comparison of epidural butorphanol tartrate and tramadol hydrochloride for postoperative analgesia using CSEA technique. Int J Res Med 2015;4(1):1-6.

3. Tanaka E, North RA. Opioid actions on rat anterior cingulate cortex neurons in vitro. J Neurosci 1994;14(3):1106-1113. DOI: 10.1523/ JNEUROSCI.14-03-01106.1994.

4. Kaur J, Bajwa SJS. Comparison of epidural butorphanol and fentanyl as adjuvants in the lower abdominal surgery: A randomized clinical study. Saudi J Anaesth 2014;8(2):167-171. DOI: 10.4103/1658354X.130687.

5. Bromage PR, Camporesi EM, Durant PA, et al. Nonrespiratory side effects of epidural morphine. Anesth Analg 1982;61(6):490-495. DOI: 10.1213/00000539-198206000-00002.

6. Sultan P, Gutierrez MC, Carvalho B. Neuraxial morphine and respiratory depression. Drugs 2011;71(14):1807-1819. DOI: 10.2165/11596250000000000-00000.

7. Rosow CE. Butorphanol in perspective. Acute Care 1988; 12(Suppl 1):2-7.

8. Von Korff M, Jensen MP, Karoly P. Assessing global pain severity by self-report in clinical and health services research. Spine (Phila Pa 1976) 2000;25(24):3140-3151.

9. Massad I, Mahafza T, Abu-Halawah S, et al. Postoperative pain is undertreated: results from a local survey at Jordan University Hospital. East Mediterr Heal J 2013;19(5):485-489. DOI: 10.26719/2013.19.5.485.
10. Buggy DJ, Smith G. Epidural anaesthesia and analgesia: better outcome after major surgery? Growing evidence suggests so. BMJ 1999;319(7209):530-531. DOI: 10.1136/bmj.319.7209.530.

11. Abboud TK, Afrasiabi A, Zhu J, et al. Epidural morphine or butorphanol augments bupivacaine analgesia during labor. Reg Anesth 1989;14(3):115-120.

12. Abboud TK, Zhu J, Afrasiabi A, et al. Epidural butorphanol augments lidocaine sensory anesthesia during labor. Reg Anesth 1991;16(5): 265-267.

13. Mittapally S, Aerram S. Efficacy of bupivacaine and fentanyl v/s bupivacaine and butorphanol in postoperative pain relief. Sch J Appl Med Sci 2016;4:3954-3956. DOI: 10.21276/sjams.2016.4.11.19.

14. Praveen PVVSB, Chandra V, Konda R, et al. A prospective, randomized, double blind, comparative study of intramuscular nalbuphine hydrochloride, butorphanol tartrate and pentazocine lactate for post-operative. Int J Basic Clin Pharmacol 2016;5(6):2326-2331. DOI: 10.18203/2319-2003.ijbcp20164049.

15. Singha D, Singh J, Bhandari S. A comparative analysis of epidural butorphanol and fentanyl for post-operative analgesia in lower abdominal surgeries. Int J Med Res Prof Orig 2016;2(2):25-32.

16. Fatima N, Singh N, Singh LK, et al. Comparative study of the effect of dexmedetomidine and butorphanol as epidural adjuvants in abdominal hysterectomy under intrathecal levobupivacaine anesthesia. J Med Soc 2016;30(3):166. DOI: 10.4103/0972-4958.191183.

17. Kar P. A comparative study between epidural butorphanol with bupivacaine and bupivacaine alone for intra-operative and postoperative analgesia in lower limb orthopaedic surgeries. Int J Res Med Sci 2016;4(10):4251-4255. DOI: 10.18203/2320-6012.ijrms20163101. 